\title{
Gastrografin as an alternative booster to sodium phosphate in colon capsule endoscopy: safety and efficacy pilot study
}

Authors

Institutions

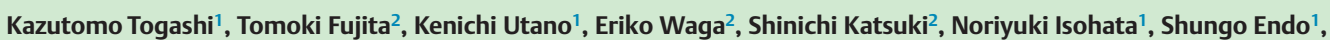
Alan K. Lefor ${ }^{3}$

${ }^{1}$ Department of Coloproctology, Aizu Medical Center, Fukushima Medical University, Aizuwakamatsu-city, Japan

${ }^{2}$ Department of Gastroenterology, Otaru Ekiseikai General Hospital, Otaru, Japan

${ }^{3}$ Department of Surgery, Jichi Medical University, Shimotsuke, Japan submitted

13. March 2015

accepted after revision

11. August 2015

\section{Bibliography}

DOI http://dx.doi.org/

10.1055/s-0034-1393075

Published online: 23.9.2015

Endoscopy International Open

2015; 03: E659-E661

(c) Georg Thieme Verlag KG

Stuttgart $\cdot$ New York

E-ISSN 2196-9736

\section{Corresponding author \\ Kazutomo Togashi, MD}

Department of Coloproctology Aizu Medical Center Fukushima Medical University

Aizuwakamatsu-city

Fukushima-prefecture

965-0024 Japan

Fax: +81-242-752568

togashik@fmu.ac.jp
Background and study aims: Sodium phosphate is a key component of bowel preparation regimen for colon capsule endoscopy (CCE), but may cause serious complications. The aim of this study is to evaluate the use of Gastrografin, substituted for sodium phosphate, in CCE bowel preparation.

Patients and methods: In total, 29 patients (median age 64 years; 23 females) underwent CCE, covered by the national health insurance system of Japan. All had a history of laparotomy and/or previously incomplete colonoscopy. On the day before examination, patients ingested $1 \mathrm{~L}$ of polyethylene glycol + ascorbic acid with $0.5 \mathrm{~L}$ of water in the evening, and again the same laxative on the morning of examination. After capsule inges-

\section{Introduction \\ $\nabla$}

Sodium phosphate (NaP) is a key component of the standard bowel preparation regimen for colon capsule endoscopy (CCE, PillCom ${ }^{\circledR}$ COLON2 Capsule Endoscopy: Medtronic, Minneapolis, MN, USA), particularly to cause excretion of the capsule endoscope while the battery is still functioning. Previous reports have demonstrated that other laxatives including polyethylene glycol and magnesium citrate were not effective for excreting the capsule endoscope [1-3]. However, the use of NaP is limited due to the possibility of causing severe adverse events, e.g. acute phosphate nephropathy, acute renal failure, hypertension, or mineral imbalance $[4,5]$. The U.S. Food and Drug Administration issued a warning to restrict its purchase without a prescription in 2008. In Japan, administration of $\mathrm{NaP}$ for hypertensive patients 64 years or older has been contraindicated since February 2012. Therefore, an alternative to $\mathrm{NaP}$ is needed to perform CCE safely with a high completion rate.
* This pilot study was conducted at two medical centers (Aizu and Otaru) in Japan. tion, $50 \mathrm{~mL}$ of Gastrografin diluted with $0.9 \mathrm{~L}$ of magnesium citrate was administered, and then repeated after 1 hour.

Results: The capsule excretion rate was 97\% (28/ $29)$. The median colon transit time was 2 hours 45 minutes and rapid transit (<40 minutes) through the colon occurred in one patient (3.4\%). Bowel cleansing level was adequate in $90 \%$ of patients. The polyp ( $\geq 6 \mathrm{~mm}$ ) detection rate was $52 \%$. Diluted Gastrografin was well tolerated by patients. No adverse events occurred.

Conclusion: Gastrografin can be an alternative to sodium phosphate in CCE bowel preparation regimen.

According to the latest report investigating the performance of CCE compared with CT colonography [6], Gastrografin (the water-soluble iodinated radiopaque oral contrast medium) was first used for the CCE regimen, because Gastrografin is generally used as part of the CT colonography regimen for "fecal tagging" [7]. In our experience, a CCE regimen using Gastrografin without NaP, achieved a very high capsule excretion rate. The gastrointestinal transit time as well as the colon transit time with this bowel preparation regimen were comparable to that of CCE regimens using a $\mathrm{NaP}$ booster. In this paper, we report the results of a pilot study using Gastrografin as a booster in the bowel preparation regimen for CCE.

\section{Patients and methods}

From June 2014 to November 2014, 29 patients in two tertiary care hospitals (Aizu Medical Center and Otaru Ekiseikai General Hospital) underwent CCE, covered by the national health insurance system of Japan. To be eligible for insurance payment for colon capsule endoscopy, the patient must meet standardized indications for colon screening 
as well as one of the following conditions including: a previously incomplete colonoscopy, a previous laparotomy, or being unable to undergo colonoscopy due to some organic abnormality. Patient demographic data is shown in Table 1. The median age was 64 years and eight patients were older than 75 years. Most patients (79\%) were female. All had a history of laparotomy and/ or previously incomplete colonoscopy. Patients with a history of bowel resection were not included. Sixteen patients (55\%) underwent the procedure to evaluate a positive fecal immunochemical test.

Details of the CCE regimen are shown in $\bullet$ Table 2. Bowel preparation started from breakfast on the day before examination. Patients had low residue meals at breakfast and lunch, and drank 1 L of polyethylene glycol (PEG) plus ascorbic acid with $0.5 \mathrm{~L}$ of water in the evening, and $10 \mathrm{~mL}$ of sodium picosulfate hydrate at bedtime. On the morning of the examination, patients again drank $1 \mathrm{~L}$ of PEG plus ascorbic acid with $0.5 \mathrm{~L}$ of water. After capsule ingestion, $50 \mathrm{~mL}$ of Gastrografin diluted with $0.9 \mathrm{~L}$ of magnesium citrate was administered to facilitate capsule excretion. Gastrografin with magnesium citrate was given again after 1 hour. If the capsule was not expelled within 4 hours of ingestion, $50 \mathrm{~mL}$ of Gastrografin with water (by mouth) and/or a bisacodyl suppository were added. The capsule excretion rate within the duration of battery life, gastrointestinal transit time, colon transit time, bowel cleansing, and yield of colon polyp identification were measured. Bowel cleansing was rated on a four-point scale (poor, fair, good, excellent) to describe the preparation of the colon at the time of the capsule endoscopy $[8,9]$.

\section{Results}

$\nabla$

The capsule excretion rate was $97 \%$ (28/29). The one patient who did not expel the capsule within the duration of battery life was a 51-year-old man with severe diverticular disease of the sigmoid colon. Gastrointestinal transit time, colon transit time, bowel cleansing, and yield of colon polyps are shown in $\bullet$ Table 3. Median colon transit time was 165 minutes and rapid transit $(<40$ minutes) through the colon occurred in one patient $(3.4 \%, 1 / 29)$. Bowel cleansing was adequate (excellent/good) in $90 \%$ of patients. The polyp ( $\geq 6 \mathrm{~mm}$ ) detection rate was $52 \%$. All patients followed our regimen and diluted Gastrografin was well tolerated by all of them. No adverse events occurred in this pilot study.

\section{Discussion}

In our CCE regimen without NaP, but using Gastrografin instead, the capsule excretion rate, gastrointestinal transit time, colon transit time, and bowel cleansing were equivalent to previous reports using NaP as a booster $[6,10,11]$. Bowel cleansing was adequate in most patients, allowing a high polyp detection rate. These results indicate that Gastrografin is an alternative to $\mathrm{NaP}$ for CCE bowel preparation.

Gastrografin, known generically as diatrizoate meglumine and diatrizoate sodium, was developed as a water-soluble iodinated radiopaque oral contrast medium in 1960, and is still used in radiographic examinations because of its unique characteristics. According to the manufacturer's information [http://www.bayerhealthcare.at/de/produkte/g-1/gastrografin/index.php], the osmolality of Gastrografin is nine times as high as that of blood plasma although the osmolality of NaP could be more than 30
Table 1 Patient demographic data.

\begin{tabular}{|llc|}
\hline All patients, $\mathrm{n}(\%)$ & & $29(100)$ \\
\hline Age, years & Mean & 64.1 \\
\hline & Median & 64 \\
\hline & Range & $42-91$ \\
\hline Gender, n (\%) & Female & $23(79)$ \\
\hline & Male & $6(21)$ \\
\hline Previous laparotomy, n (\%) & Yes & $19(66)$ \\
\hline $\begin{array}{l}\text { Previous incomplete colo- } \\
\text { noscopy, } \mathrm{n}(\%)\end{array}$ & Yes & $16(55)$ \\
\hline $\begin{array}{l}\text { Indication for colonoscopy, } \\
\text { n (\%) }\end{array}$ & Positive fecal immuno- & $16(55)$ \\
\hline & chemical test & $6(21)$ \\
\hline & Post-polypectomy & $4(14)$ \\
\hline & Change in bowel habits & $2(7)$ \\
\hline & Hematochezia & $1(3)$ \\
\hline
\end{tabular}

\begin{tabular}{|c|c|c|}
\hline Day & Hour & Action \\
\hline \multirow{3}{*}{$\begin{array}{l}\text { Day before } \\
\text { exam }\end{array}$} & All day & Low-residue diet \\
\hline & Evening & Moviprep $1 \mathrm{~L}+$ water $0.5 \mathrm{~L}$ \\
\hline & Bedtime & Laxoberon $10 \mathrm{~mL}+$ water $0.2 \mathrm{~L}$ \\
\hline \multirow[t]{10}{*}{ Day of exam } & 07:00 & Moviprep $1 \mathrm{~L}+$ water $0.5 \mathrm{~L}$ \\
\hline & 09:30 & Capsule ingestion \\
\hline & & + $0.1 \%$ domperidone syrup $30 \mathrm{~mL}(30 \mathrm{mg})$ \\
\hline & & + mosapride $10 \mathrm{mg}$ \\
\hline & $10: 30$ & Magcorol P isotonic solution $0.9 \mathrm{~L}$ \\
\hline & & + mosapride $10 \mathrm{mg}$ \\
\hline & & + Gastrografin $50 \mathrm{~mL}$ \\
\hline & $11: 30$ & Magcorol P isotonic solution $0.9 \mathrm{~L}$ \\
\hline & & + Gastrografin $50 \mathrm{~mL}$ \\
\hline & $12: 30$ & Suppository (bisacodyl $10 \mathrm{mg}$ ) \\
\hline
\end{tabular}

Moviprep: polyethylene glycol + ascorbic acid; Laxoberon: sodium picosulfate hydrate; Magcorol P: magnesium citrate; Gastrografin: diatrizoate meglumine and diatrizoate sodium.

\begin{tabular}{|c|c|c|}
\hline $\begin{array}{l}\text { Capsule excretion rate within the life } \\
\text { of the battery, } n(\%)\end{array}$ & & $28(97)$ \\
\hline \multirow[t]{2}{*}{ Gastrointestinal transit time, min } & Median & 281.5 \\
\hline & Range & $105-641$ \\
\hline \multirow[t]{2}{*}{ Colon transit time, min } & Median & 165 \\
\hline & Range & $25-565$ \\
\hline \multirow[t]{4}{*}{ Bowel cleansing, $n(\%)$} & Excellent & $11(38)$ \\
\hline & Good & $15(52)$ \\
\hline & Fair & $3(10)$ \\
\hline & Poor & $0(0)$ \\
\hline \multirow[t]{3}{*}{ Polyp yield, n (\%) } & None $^{1}$ & $14(48)$ \\
\hline & $6-10 \mathrm{~mm}$ & $7(24)$ \\
\hline & $\geq 11 \mathrm{~mm}$ & $8(28)$ \\
\hline
\end{tabular}

${ }^{1}$ Includes polyps $\leq 5 \mathrm{~mm}$

times higher in preliminary calculations. Therefore, Gastrografin can work as a hyperosmotic laxative. While NaP is likely to increase the serum level of inorganic phosphate due to high phosphate level, most of the Gastrografin is not absorbed from the intestinal tract into the circulation. Overall, $\leq 2 \%$ of Gastrografin is excreted into the urine without being metabolized. These characteristics suggest that an electrolyte imbalance is less frequently 
caused by oral/anal administration of Gastrografin. Gastrografin is a safe hyperosmotic laxative, and is a booster suitable for patients at increased risk. In this pilot study, including eight patients older than 75 years, no adverse events occurred.

The latest clinical trial [12] conducted in the United States used a sulfate solution (SUPREP; Braintree Laboratories) as a booster for colon capsule endoscopy. In this large clinical trial, the median colon transit time was 75 minutes and occasionally the capsule passed through the colon within 40 minutes. Subsequently, approximately $10 \%(77 / 772)$ of subjects were excluded from the final analysis, based on the combination of poor preparation and rapid transit after enrollment. Because the sulfate solution is poorly absorbed, the osmotic effect of unabsorbed sulfate anions and associated cations causes water to be retained within the intestine, thus expelling the capsule in a short time. In the present study using Gastrografin as a booster, the median colon transit time (165 minutes) was longer than that in the clinical trial using a sulfate solution. Gastrografin booster may provide sufficient observation time for the colon. Indeed, the polyp ( $\geq 6 \mathrm{~mm}$ ) detection rate was comparably high (52\%) in the present study.

There are acknowledged limitations to this study. This is a pilot study before a clinical trial, which limits the generalizability of the results. In addition, the present study was conducted in highly selected patients with a history of laparotomy and/or previously incomplete colonoscopy, possibly limiting the ability to generalize these results to other patient groups. We acknowledge that the polyp detection with CCE has not been verified by follow-up colonoscopy, which could alter the results of the yield of colon polyps. Despite these limitations, we believe that consideration of Gastrografin as an alternative booster is of interest. In conclusion, the use of Gastrografin in the CCE bowel preparation regimen is promising. Other modifications to the protocol may influence the high capsule excretion rate. A clinical trial is warranted to compare regimens with and without Gastrografin.

\section{References}

1 Sieg A, Friedrich $K$, Sieg $U$. Is PillCam COLON capsule endoscopy ready for colorectal cancer screening? A prospective feasibility study in a community gastroenterology practice Am J Gastroenterol 2009; 104: $848-854$

2 Spada C, Riccioni ME, Hassan C et al. PillCam colon capsule endoscopy: a prospective, randomized trial comparing two regimens of preparation. J Clin Gastroenterol 2011; 45: 119-124

3 Singhal S, Nigar S, Paleti V et al. Bowel preparation regimens for colon capsule endoscopy: a review. Therap Adv Gastroenterol 2014; 7 : 115 122

4 Markowitz GS, Nasr SH, Klein P et al. Renal failure due to acute nephrocalcinosis following oral sodium phosphate bowel cleansing. Hum Pathol 2004; 35: 675-684

5 Ehrenpreis ED, Parakkal D, Semer $R$ et al. Renal risks of sodium phosphate tablets for colonoscopy preparation: a review of adverse drug reactions reported to the US Food and Drug Administration. Colorectal Dis 2011; 13: e270-e275

6 Spada C, Hassan C, Barbaro B et al. Colon capsule versus CT colonography in patients with incomplete colonoscopy: a prospective, comparative trial. Gut 2015; 64: 272-281

7 Johnson $C D$, Chen $M H$, Toledano AY et al. Accuracy of CT colonography for detection of large adenomas and cancers. NEJM 2008; 359: $1207-$ 1217

8 Eliakim R, Fireman Z, Gralnek IM et al. Evaluation of the PillCam Colon capsule in the detection of colonic pathology: results of the first multicenter, prospective, comparative study. Endoscopy 2006; 38: 963 - 970

9 Schoofs N, Deviere J, Van Gossum A. PillCam colon capsule endoscopy compared with colonoscopy for colorectal tumor diagnosis: a prospective pilot study. Endoscopy 2006; 38: 971 -977

10 Van Gossum A, Munoz-Navas M, Fernandez-Urien I et al. Capsule endoscopy versus colonoscopy for the detection of polyps and cancer. NEJM 2009; 361: 264-270

11 Rondonotti E, Borghi C, Mandelli G et al. Accuracy of capsule colonoscopy and computed tomographic colonography in individuals with positive results from the fecal occult blood test. Clin Gastroenterol Hepatol 2014; $12: 1303-1310$

12 Rex DK, Adler SN, Aisenberg J et al. Accuracy of capsule colonoscopy in detecting colorectal polyps in a screening population. Gastroenterology 2015 ; 148: $948-957$

\section{Competing interests: None}

